\title{
A multicenter survey of pediatric flexible bronchoscopy in western China
}

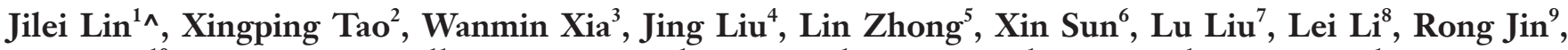 \\ Li Cheng ${ }^{10}$, Zhongtao Wang ${ }^{11}$, Zhengxiu Luo ${ }^{1}$, Qubei Li ${ }^{1}$, Enmei Liu ${ }^{1}$, Zhou Fu ${ }^{1}$, Jihong Dai ${ }^{1}$
}

${ }^{1}$ Department of Respiratory Disease, Children's Hospital of Chongqing Medical University, National Clinical Research Center for Child Health and Disorders, Ministry of Education Key Laboratory of Child Development and Disorders, Chongqing Key Laboratory of Pediatrics, Chongqing, China; ${ }^{2}$ Department of Pediatrics, Kaiyuan People's Hospital, Kaiyuan, China; ${ }^{3}$ Department of Pediatrics, Chengdu Women's and Children's Central Hospital, School of Medicine, University of Electronic Science and Technology of China, Chengdu, China; ${ }^{4}$ Department of Pediatrics, the First Affiliated Hospital of Guangxi Medical University, Nanning, China; ${ }^{5}$ Department of Respiratory Disease, West China Second University Hospital of Sichuan University, Chengdu, China; ${ }^{6}$ Department of Pediatrics, The Fourth Medical University, Xi'an, China; ${ }^{7}$ Department of Pediatrics, Urumqi Children's Hospital, Urumqi, China; ${ }^{8}$ Department of Pediatrics, Yinchuan Maternal and Child Health Hospital, Yinchuan, China; ${ }^{9}$ Department of Pediatrics, Guiyang Maternal and Child Health Hospital, Guiyang, China; ${ }^{10}$ Department of Pediatrics, Qinghai Women and Children Hospital, Xining, China; ${ }^{11}$ Department of Pediatrics, Gansu Provincial Maternity and Child-Care Hospital, Lanzhou, China

Contributions: (I) Conception and design: J Dai, Z Fu, J Lin; (II) Administrative support: J Dai, Z Fu, E Liu, Z Luo, Q Li; (III) Provision of study materials or patients: J Lin, X Tao, W Xia, J Liu, L Zhong, X Sun, L Liu, L Li, R Jin, L Cheng, Z Wang; (IV) Collection and assembly of data: J Lin, J Dai; (V) Data analysis and interpretation: J Dai, Z Fu, J Lin; (VI) Manuscript writing: All authors; (VII) Final approval of manuscript: All authors.

Correspondence to: Jihong Dai. Department of Respiratory Disease, Children's Hospital of Chongqing Medical University, 136 Zhongshan 2nd Rd, Lianglukou St, Yuzhong Dist, Chongqing 400014, China. Email: danieljh@163.com.

Background: This study aims to investigate the current use of pediatric flexible bronchoscopy (PFB) in western China.

Methods: A cross-sectional survey was conducted in western China between January 1, 2018 to December 30, 2018. Fifty-four centers were invited to answer a questionnaire for seeking information about performance of PFB. The data collected were analyzed to investigate the current status of western China, and hierarchical cluster analysis was conducted to identify developmental level of PFB of cities.

Results: Forty-seven centers were included in analysis. A total of 22,585 flexible bronchoscopies were carried out in the participating centers from January 1, 2018 to December 30, 2018. Eight centers $(17.0 \%)$ performed more than 1,000 pediatric flexible bronchoscopies for children, but 20 centers $(42.6 \%)$ performed less than 100 . The median proportion of systematic and professional trained physicians in a single team was 50\%, and the pooled rate was 59\% (95\% CI, 47-70\%). Only 10, 8 and 11 centers performed balloon dilatation, thermal ablation and cryoablation, respectively. Obvious cough was the most frequent complication after the PFB procedure, the pooled rate is 24\% (95\% CI, 18-29\%). No one died during and after the PFB procedure. Hierarchical cluster analysis showed that the development of PBF in western China varies, and Chongqing might be the most developed area in PFB use in western China.

Conclusions: Flexible bronchoscopy in children is now a mature and safe procedure, while the development of PFB varies in western China, especially for the advanced bronchoscopic intervention.

Keywords: Flexible bronchoscopy; survey; western China

Submitted Aug 17, 2020. Accepted for publication Nov 13, 2020.

doi: $10.21037 /$ tp-20-244

View this article at: http://dx.doi.org/10.21037/tp-20-244

^ ORCID: 0000-0003-1920-9317. 


\section{Introduction}

With the development of technology, bronchoscopes are available in a range of sizes and lengths, enabling bronchoscopic practice wildly used in children, even neonates (1-3). Bronchoscopic procedures have not been standardized yet among hospitals in China, though Chinese guidelines on pediatric flexible bronchoscopy (PFB) have been published in 2009 (4) and updated in 2018 (5). Compared with developed countries and eastern China, western China is regarded as a developing area in economy and medical service. Therefore, the development of PFB keeps slow in western China. In order to improve the skills of $\mathrm{PBF}$ and standardize the procedure, Pediatric Intervention Pulmonology of Collaborative Group was established in western China (PIPCG) with one provincelevel municipality (Chongqing) and nine provinces (Shanxi, Gansu, Qinghai, Xinjiang, Sichuan, Yunnan, Guizhou, Guangxi and Ningxia) in 2019. Previous studies reported surveys on bronchoscopy were performed in many countries (6-9), while there is no large-scale survey of PFB in China till now. Thus, it is essential to understand the current status of PFB in western China and provide assistance to different area by improving the development of PFB.

Considering the factors above, a questionnaire was designed and sent to pediatric centers by PIPCG in western China. The purpose of this survey was to investigate the current status of PFB use in western China. We present the following article in accordance with the STROBE reporting checklist (available at http://dx.doi.org/10.21037/tp-20244).

\section{Methods}

The study was conducted in accordance with the Declaration of Helsinki (as revised in 2013). Ethical approval for this study was not required. As our study was mainly aimed at the development of bronchoscopy in west China, the objects of our research are pediatric centers, we did not collect the specific information of patients.

To collect information about the up-to-date development of PFB in western China, a questionnaire was sent to 9 main member units of PIPCG via WeChat (a chat application) in March 2019. The 9 units then contacted the respiratory or pediatric centers performing PFB in their relevant provinces or neighboring regions and recruited them for the survey. The detailed information of self-designed questionnaire is shown in Table S1.

\section{Statistical analysis}

The continuous variables were presented as median with range because most of variables were not normally distributed, while the categorical variables were presented as rate and incidence. The rates of conducting PFB stratified according to distinct indications and adverse events were pooled, with random effects model (10). To identify developmental level of PFB of cities, we conducted a hierarchical cluster analysis (HCA). Similarity between cases was computed with the Gower distance and complete linkage was used as the agglomeration procedure (11). All analyses were performed using R (version 3.61 for Windows). A $\mathrm{P}$ value $<0.05$ was considered statistically significant, and all tests were two-sided.

\section{Results}

The questionnaires were sent to 54 pediatric centers in western China, all of the questionnaires were answered and returned. The centers were contacted again to provide the missing information by E-mail or telephone when answers were incomplete. Seven of 54 centers were dropped out from the final analysis because one center provided obviously wrong information without any explanation, two centers started developing PFB in 2019, one in August 2018, and the medical equipment of flexible bronchoscopy was just introduced but not applied to clinic till receiving the questionnaire in another three centers. All the cities included in analysis were listed in Table S2.

\section{Overview of participating respiratory or pediatric centers}

In this survey, a total of 22,585 flexible bronchoscopies were carried out in the participating centers from 11 provinces in western China from January 1, 2018 to December 30, 2018 (Figure 1). The median number of PFB was 158 in a single center in one year. 8 centers $(17.0 \%)$ performed more than 1,000 pediatric flexible bronchoscopies for children, but 20 centers $(42.6 \%)$ performed less than 100 . The median maximum age of participating children was 14 years old, and median minimum age was 27 days old (Table S3).

\section{Instruments}

China-made equipment was used in only 7 centers (14.9\%), while Japanese bronchoscopies (Olympus, FUJINON and PENTAX) were applied in 46 centers (97.9\%). Detailed 


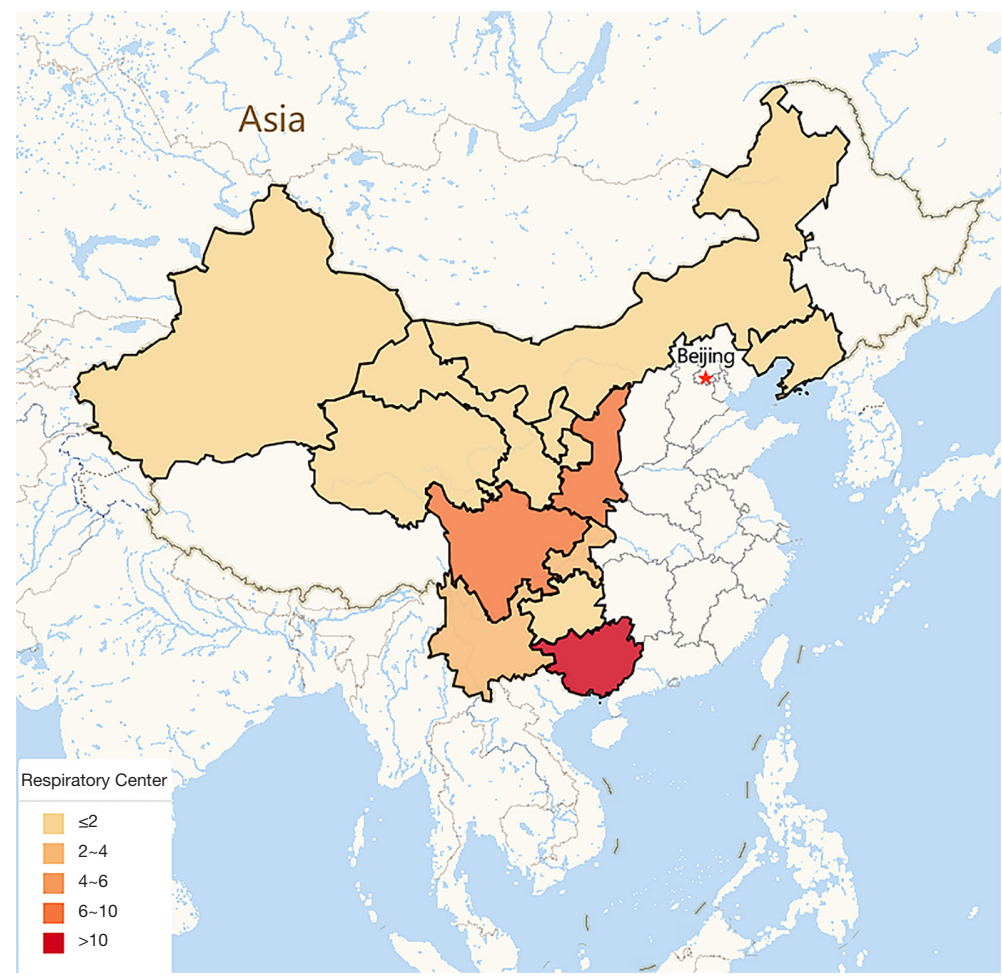

Figure 1 The included provinces of this study.

information is shown in Table S3. The instruments of balloon dilatation, thermal ablation and cryoablation were equipped in 10, 8 and 11 centers, respectively (Table S4). One center had instrument of transbronchiallung biopsy (TBLB).

\section{Personnel}

A team of PFB usually consists of physicians, nurses and anesthesiologists. This survey showed the median number of physicians was 5 and the median number of nurses was 4 in a team. Only 24 teams $(51.1 \%)$ were staffed with anesthesiologists, with a median number of one. All physicians in 5 centers (10.6\%) didn't receive systematic and professional training in a qualified institution, the median proportion of trained physicians in a single team was $50 \%$, and the pooled rate was $59 \%$ (95\% CI, $47-70 \%)$ with significant heterogeneity $\left(\mathrm{I}^{2}=65 \%\right)$ (Figure $\mathrm{S} 1$ ). The information of physicians' professional titles and educational degrees is shown in Table 1. The median number of participating physicians and nurses are both two, but anesthesiologists took part in the bronchoscopic procedure in only 24 centers (51.1\%) (Table 1).

\section{Sites of PFB}

In this survey, endoscopy suite, ward, ICU, operating room and bronchoscopy room were main sites for performing PFB (Table S3). Most centers (79.2\%) had performed bronchoscopies in more than one site. Twenty-nine centers $(61.7 \%)$ and 31 centers $(66.0 \%)$ reported that they performed bronchoscopies in endoscopy suite and ICU, respectively. Bronchoscopies were carried out in a designated bronchoscopy suite in only 2 centers.

\section{Patient preparation}

Patients are required to complete some examinations before bronchoscopy including blood tests $(100 \%)$, radiological image (97.9\%) and pulmonary function test (40.4\%) (Table S5).

\section{Examination of acquired sample}

Bronchoalveolar lavage fluid (BALF) was tested in 45 centers $(95.7 \%)$, all of which performed bacterial culture, while the routine tests (cytology test) for BALF were carried out in 38 centers (80.9\%). Detection of pathogen by ELISA 
Table 1 Characteristics of team members of bronchoscopy in centers

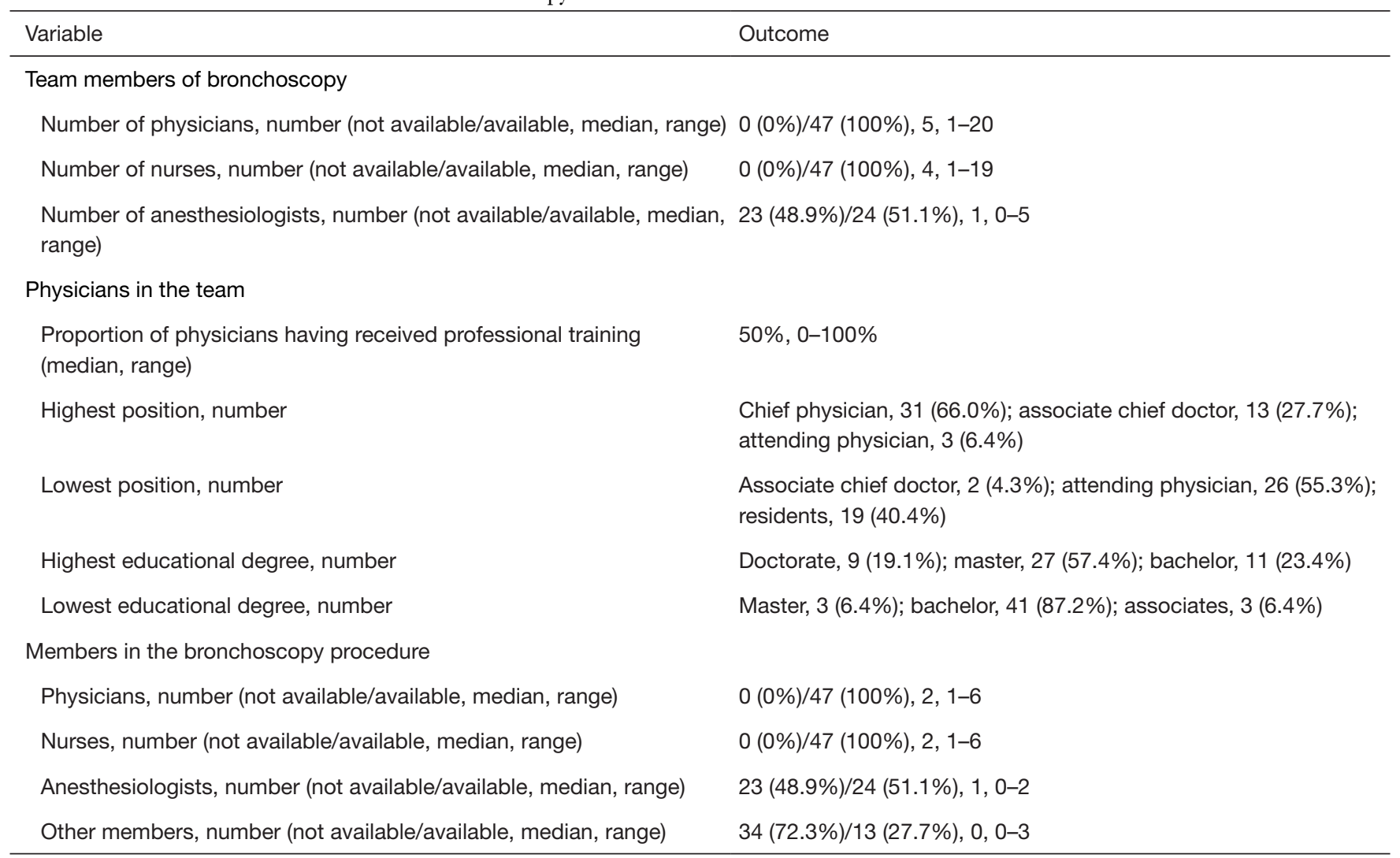

and PCR were performed in 29 centers (61.7\%). Twentysix centers $(55.3 \%)$ could perform the histopathological examination for bronchial mucosa. Only 1 center could perform the histopathological examination for lung tissue acquired by bronchoscopy.

\section{Sedation/anesthesia and first-aid equipment}

The preferences of sedation/anesthesia in centers are shown in Table S6. In this survey, local anesthesia combined with conscious sedation was applied in 30 centers $(63.8 \%)$, while intravenous anesthesia was applied in 26 centers (55.3\%). Most centers have two or more types of anesthesia for bronchoscopy. Midazolam was chosen as anesthetics in most centers (95.8\%), while lidocaine was chosen in 37 centers (77.1\%). Propofol and fentanyl was applied in 15 centers (31.9\%) and 21 centers (44.7\%), respectively. All centers prepared oxygen, ambu bags and electrocardiogram monitors during the whole procedure, and 46 centers (97.9\%) prepared suction apparatus and tracheal cannula as backup. Defibrillator and ventilator were equipped in 34 centers $(72.3 \%)$ and 35 centers $(74.5 \%)$, respectively. The detailed information of equipment is shown in Table S6.

\section{Interventional bronchoscopy}

The detailed information of bronchoscopic intervention is shown in Table S4. In this survey, the traditional pediatric bronchoscopic interventions such as bronchoalveolar lavage (BAL), transbronchial injection and bronchoscopic forceps were undertaken widely in western China. BAL, bronchoscopic injection, bronchoscopic brushing, bronchoscopic forceps were performed in 46 (97.9\%), 44 (93.6\%), $26(55.3 \%)$ and $32(68.0 \%)$ centers, respectively. There were 5 centers performing more than $1000 \mathrm{BAL}$ in one year, and more than 1,000 bronchoscopic injection were completed in 4 centers. The result of this survey showed that advanced bronchoscopic interventions were performed at small scales in western China up to today. There are 10 centers performing balloon dilatation, 8 centers performing thermal ablation, and 11 centers performing cryoablation. It is noteworthy that TBLB 
Table 2 Indication of flexible bronchoscopy and meta-analysis for proportion of disease

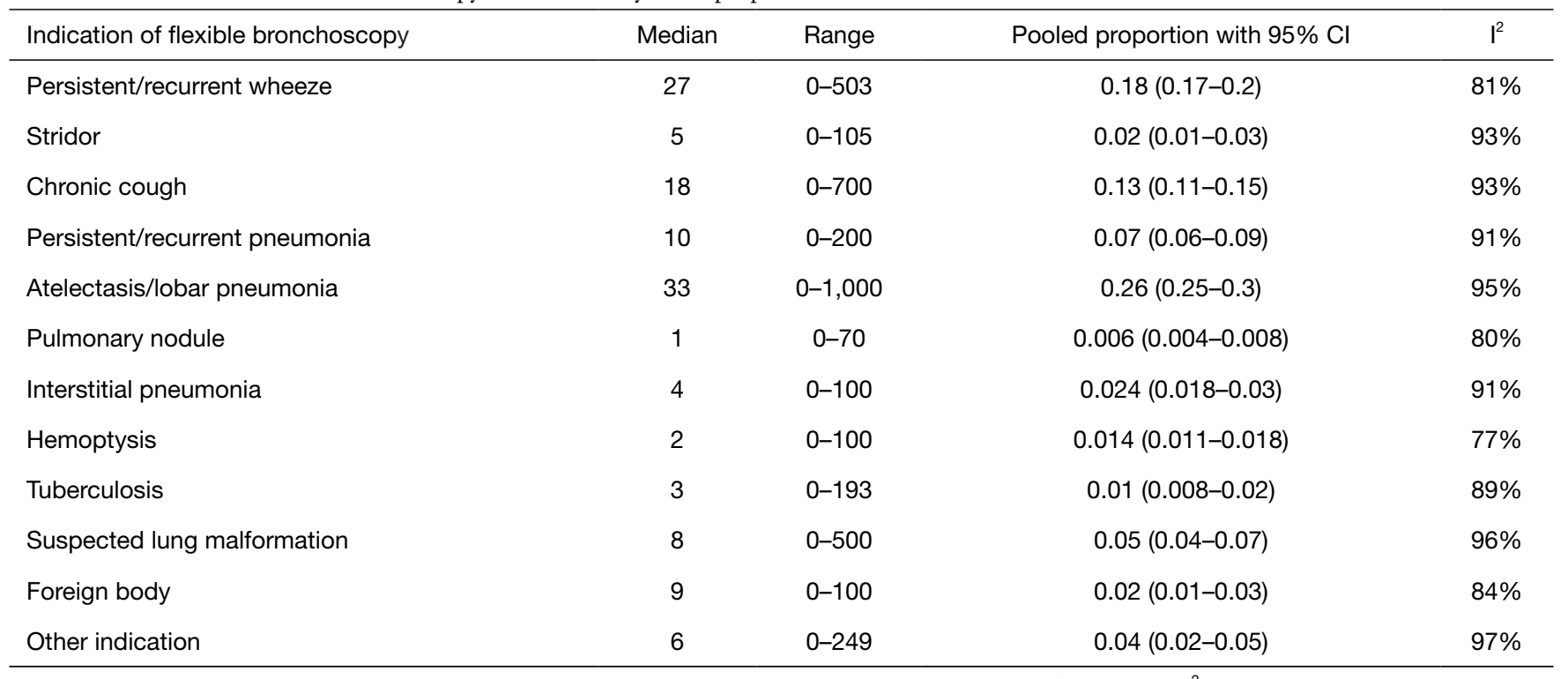

Median: the median number of participant in centers; pooled proportion performed by random effect model; $I^{2}$ is for statistical heterogeneity.

were tentatively applied to clinic in one center we studied, and there was no center performed stent placement. Furthermore, the number of participating patients with these advanced bronchoscopic interventions was small in performing centers.

\section{Indications}

All centers were asked to list the indications for PFB with specific number of patients in this survey. However, some centers provided the approximate number of indications due to the large number of patients without accurate statistics. The information about indications is shown in Table 2. We counted the proportion of every kind of indication in every center. Because the number of patients were not accuracy in some centers, we pooled the proportions using random effect model. Atelectasis/lobar pneumonia was the most frequent indication for PFB (rate 26\%, 95\% CI, 25-30\%), persistent/recurrent wheeze and chronic cough were common in every center with pooled rate of $18 \%$ (95\% CI, $17-20 \%)$ and $13 \%$ (95\% CI, 11-15\%), respectively. Other kinds of indications for bronchoscopy such as stridor, tuberculosis, pulmonary nodule, hemoptysis and foreign body aspiration were rare in this survey.

\section{Complications}

The data of complications are shown in Table 3. However, some centers also provided the approximate number of complications due to the large number of patients without accurate statistics. the most frequent complication was decreased $\mathrm{O}_{2}$ saturation/hypoxia in the $\mathrm{PFB}$ procedure, followed by bleeding. In fact, 40 centers reported hypoxia or decreased $\mathrm{O}_{2}$ saturation and 37 reported bleeding. The pooled incidence of hypoxia or decreased $\mathrm{O}_{2}$ saturation was $4 \%$ (95\% CI, 3-6\%), while the rate of bleeding was $2 \%(95 \%$ CI, $1.3-2.7 \%)$ with significantly statistical heterogeneity $\left(\mathrm{I}^{2}=85 \%\right)$. Only 2 centers reported arrhythmia and pneumothorax or submediastinal emphysema, but these complications happened on no more than 3 children in each center. Obvious cough was the most frequent complication after the PFB procedure, the pooled rate is $24 \%$ (95\% CI, $18-29 \%)$. Fever ranked second with pooled rate of $4 \%(95 \%$ CI, 3-5\%). There was no one died during and after the $\mathrm{PFB}$ procedure.

\section{The level of development of PFB in cities}

There were 32 cities included in this survey, the number of physicians and patients and advanced bronchoscopy were supposed to contribute to the level of development of PFB. We used the average number of physicians and patients if there were two or more centers in one city. We divided these cities into four levels according to the HCA (Figure 2). It showed that the development of PBF in western China varies, and Chongqing might be the most developed in $\mathrm{PFB}$ 
Table 3 The adverse events during and after flexible bronchoscopy

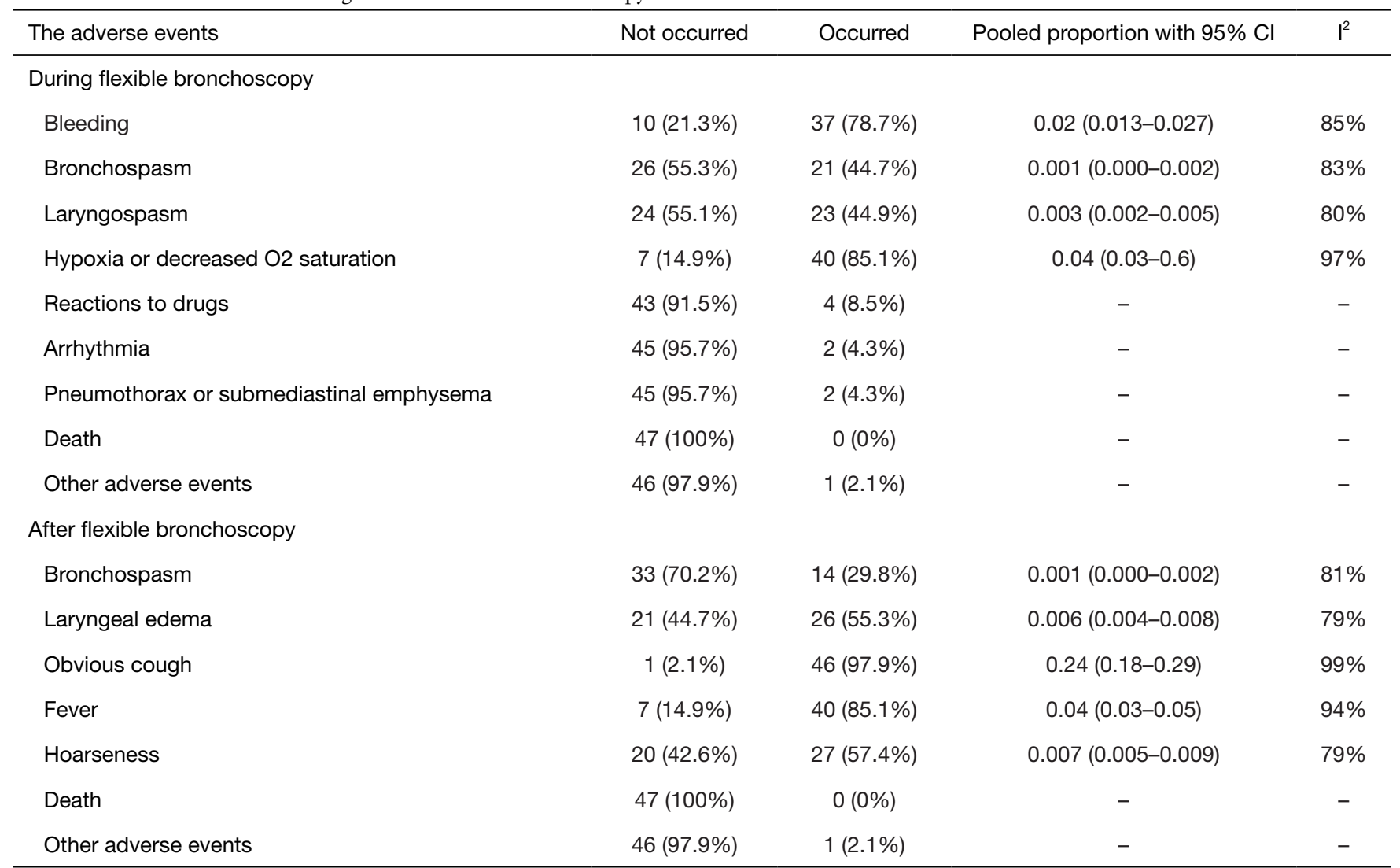

Pooled proportion performed by random effect model; $\mathrm{I}^{2}$ is for statistical heterogeneity, - is due to the limited data for meta-analysis.

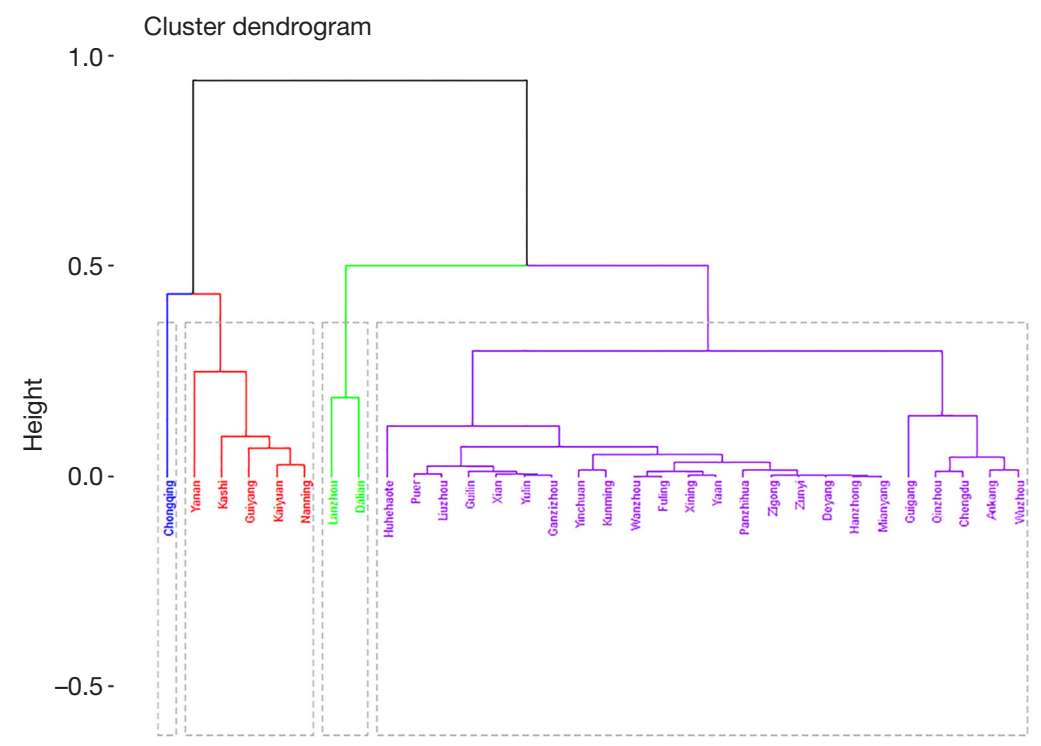

Figure 2 Hierarchical cluster analysis of these included cities. 
use in western China.

\section{Discussion}

This is the first multicenter survey which was forwarded to the pediatric or pediatric respiratory centers in western China. All of 54 questionnaires were completed and returned with no incentives offered to the centers. Finally, 47 centers were included in analysis. Although lots of pediatric respiratory centers in western China which perform PFB were included in this survey. However, the participating centers were screened by the 9 main member units of which they were familiar with.

The meta-analysis shows the proportion of physicians receiving professional training is only 59\% (95\% CI, $47-70 \%)$. The results showed that the qualification training of pediatric bronchoscopy is defective in China, but some efforts have been made by government and medical organizations. The Chinese medical doctor association have assigned 11 centers as the formal training bases, the training of pediatric bronchoscopy will be more standard in future. Flexible instruments were used in 47 centers, while the majority of centers used flexible instruments made in Japan. The survey shows that endoscopy centers and ICU wards are the most common sites for PFB in western China, some centers also performed flexible bronchoscopies in general wards and operating rooms. However, the bronchoscopies were carried out in dedicated bronchoscopic sites in only 2 centers. With the increase of medical cost, it is necessary to explore new management for hospital to reduce the cost of medical equipment management. Therefore, endoscopy centers were established for the similar standard management of all of endoscopic instruments, it can save costs and increase cooperation among different departments. Endoscopy center can manage all endoscopic equipment in disinfection and protection. When the endoscopic equipment is purchased, we can negotiate with the company to get a lower price. Moreover, when the endoscopic equipment is damaged, we can repair them at a lower price.

The indications for PFB were various at different centers and were not consistent with previous literature $(7,11)$. In order to investigate the prevalence of indications for PFB in western China, the proportions of sorts of indications in each center were pooled. The results of meta-analysis show that atelectasis/lobar pneumonia, persistent/recurrent wheeze and chronic cough are the major indications for PFB in western China. However, significant heterogeneity was obtained, which may be attributed to various proportions of indications in each center.

Our study shows that BAL and transbronchial injection were performed in the vast majority of participating centers. Examinations of BALF including culture could be carried out. Nowadays, lots of special techniques such as balloon, basket, laser, and cryoprobe are available, while advanced interventional pediatric bronchoscopy was less commonly performed in western China. There are a few of centers performing balloon dilatation, thermal ablation and cryoablation for children. Only one center developed TBLB, and no center performed stent placement. Compared with the pediatric bronchoscopy in Europe, efforts should be taken in advanced interventional pediatric bronchoscopy in western China on the premise of standard skills and proper patient selecting criteria.

The results of this survey showed that we need pay more attention to the security of the procedure of PFB. Nearly half of the centers do not have anesthesiologists taking part in the procedure. The most frequent anesthesia medicine are midazolam and lidocaine, while fentanyl is the most popular medicine in general anesthesia. Not all centers prepare defibrillator during procedure although arrhythmia occurred in 2 centers.

There are complications reported in centers during and after the PFB procedure. In order to investigate the incidence of complications in western China, we pooled the proportion of complications of each center. Among the side effects, decreased $\mathrm{O}_{2}$ saturation or hypoxia and bleeding are the most frequent during the procedure. After the PFB procedure, obvious cough and fever happen most frequently. It seems that side effects occur more in centers with lower level development of PFB, though it is difficult to find a statistical correlation between them. The survey shows that nobody died due to $\mathrm{PBF}$ procedure. To conclude, $\mathrm{PBF}$ is safe, but the professional training and more practice were required in a real-world environment because the complications may cause the life threaten.

\section{Conclusions}

This multicenter survey of pediatric respiratory centers provides detailed information which leads to a better understanding of the current status of PFB in western China. In general, PBF is safe, but strengthened personnel training and standardized procedure are needed. The level of development of advanced diagnostic and therapeutic technologies needs improving in western Chinese pediatric respiratory centers. 


\section{Acknowledgments}

We thank Prof. Chen Meng from Qilu Children's Hospital of Shandong University, Prof. Fanzheng Meng from the first Bethune Hospital of Jilin University, and Prof. Lei Chi from Dalian Children's Hospital of Dalian Medical University.We thank the effort of modification for the development of pediatric respiratory endoscopy of Pediatric respiratory endoscopy Committee of endoscopist branch of Chinese Medical Doctor Association, Pediatric interventional respiratory disease group of minimally invasive branch of China maternal and Child Health Association, and Bronchoscopy cooperation group, respiratory group of Chinese Medical Association. We appreciate the resource provision of Haizhen Wang, Yong Rao, Qi Wang, Zhengzhen Tang, Shenglin Yang, Chang Su, Yong Xie, Zheng Liang, Dejun Li, Mincai Ding, Zhijun Tan, Jinquan Lao, Xiaoxiang Yang, Lingli Lan, Li Cheng, Chonggang Huang, Haiqin La, Yadi Song, Jinglin Chen, Zhao Ma, Jianfeng Luo, Huajie Wu, Lina Chen, Li Qiu, Cheng Xia, Xueqiong Zhang, Li Wu, Xuezu Zhang, Zhilang Cao, Xinfa Chen, Gui Liao, Jiawu Yang, Xiaojun Li, Yan Li, Xun Chen, Min Rao, Junjie Ning, Yan Li, Xueyu Wang, Bo Wen, Liping Wang, Hongdong Liu, Min Wang, Yingxuan Huang, Ming Liang, Yong Lv. We also appreciate the effort of modification for the development of bronchoscopy by Pediatric Intervention Pulmonology of Collaborative Group in western China.

Funding: This work was supported by Zhou Fu: Chongqing elite-innovation leader.

\section{Footnote}

Reporting Checklist: The authors have completed the STROBE reporting checklist. Available at http://dx.doi. org/10.21037/tp-20-244

Data Sharing Statement: Available at http://dx.doi. org/10.21037/tp-20-244

Conflicts of Interest: All authors have completed the ICMJE uniform disclosure form (available at http://dx.doi. org/10.21037/tp-20-244). All authors report grants from Zhou Fu: Chongqing elite-innovation leader (Grant No. 020160), outside the submitted work.

Ethical Statement: The authors are accountable for all aspects of the work in ensuring that questions related to the accuracy or integrity of any part of the work are appropriately investigated and resolved. The study was conducted in accordance with the Declaration of Helsinki (as revised in 2013). Ethical approval for this study was not required. Because our study was mainly aimed at the development of bronchoscopy in west China, the objects of our research are pediatric centers, we did not collect the specific information of patients.

Open Access Statement: This is an Open Access article distributed in accordance with the Creative Commons Attribution-NonCommercial-NoDerivs 4.0 International License (CC BY-NC-ND 4.0), which permits the noncommercial replication and distribution of the article with the strict proviso that no changes or edits are made and the original work is properly cited (including links to both the formal publication through the relevant DOI and the license). See: https://creativecommons.org/licenses/by-nc-nd/4.0/.

\section{References}

1. Faro A, Wood RE, Schechter MS, et al. Official American Thoracic Society technical standards: flexible airway endoscopy in children. Am J Respir Crit Care Med 2015;191:1066-80.

2. Jayaraj AK, Jayaraj PK, Murugesh M, et al. Tracheal Foreign Body Removal Using Flexible Bronchoscope in a Pediatric Patient. A Novel Approach. 2017;196:1071-2.

3. Sachdev A, Chhawchharia R, Gupta D, et al. Flexible Fiberoptic Bronchoscopy Directed Interventions in Neonatal Intensive Care Unit. Indian Pediatr 2019;56:563-5.

4. Pediatric bronchoscopy collaboration group, respiratory group, pediatric Pediatric Society of Pediatrics. pediatric bronchoscopy guidelines (2009 Edition)[translation into English]. Chinese Journal of Pediatrics 2009; 47:740.

5. Expert group of pediatric respiratory endoscopy diagnosis and treatment technology of talent exchange service center of National Health Commission. Guidelines for Chinese pediatric flexible bronchoscopy (2018 Edition) [translation into English]. Chinese Journal of Practical Pediatrics 2018;33:983-9.

6. Barbato A, Magarotto M, Crivellaro M, et al. Use of the paediatric bronchoscope, flexible and rigid, in 51 European centres. Eur Respir J 1997;10:1761-6.

7. Midulla F, de Blic J, Barbato A, Bush A, et al. Flexible 
endoscopy of paediatric airways. Eur Respir J 2003;22:698-708.

8. Honeybourne D, Neumann CS. An audit of bronchoscopy practice in the United Kingdom: a survey of adherence to national guidelines. Thorax 1997;52:709-13.

9. Barnett AM, Jones R, Simpson G. A Survey of Bronchoscopy Practice in Australia and New Zealand. J Bronchology Interv Pulmonol 2016;23:22-8.

Cite this article as: Lin J, Tao X, Xia W, Liu J, Zhong L, Sun X, Liu L, Li L, Jin R, Cheng L, Wang Z, Luo Z, Li Q, Liu E, Fu Z, Dai J. A multicenter survey of pediatric flexible bronchoscopy in western China. Transl Pediatr 2021;10(1):83-91. doi: 10.21037/ tp-20-244
10. Desikan P, Khan Z. Prevalence of hepatitis B and hepatitis $\mathrm{C}$ virus co-infection in India: A systematic review and meta-analysis. Indian J Med Microbiol 2017;35:332-9.

11. Schramm D, Yu Y, Wiemers A, et al. Pediatric flexible and rigid bronchoscopy in European centersAvailability and current practice. Pediatr Pulmonol 2017;52:1502-8. 